\title{
Family Caregiving in Dementia: The Asian Perspective of a Global Problem
}

\author{
Sally Wai-Chi Chan \\ Alice Lee Centre for Nursing Studies, Yong Loo Lin School of Medicine, National University of Singapore, Singapore
}

\section{Key Words}

Dementia $\cdot$ Family caregivers $\cdot$ Burden $\cdot$ Asian

\begin{abstract}
Background/Aims: With an increasing life expectancy, the number of people suffering from dementia globally will rise to about 115.4 million by 2050 . It is projected that in the $21 \mathrm{st}$ century, the majority of the world's older people will be living in Asia. This paper examines the global issues of family caregiving for older persons with dementia from an Asian perspective. Methods: Literature review of published empirical studies from 1990 to October 2010. Results: A full understanding of the specific way that culture could influence family caregivers' burdens may play an important part in the development of a holistic model for family-centered care. Well-designed psychoeducation interventions or multicomponent interventions could help in reducing caregivers' burden. With socio-economical and demographical changes, families need to be supported when taking on the caregiving role and be offered alternatives of care. Conclusion: There is a need for future trials with more rigorous randomization and longitudinal follow-up to assess family caregiver burdens at different illness stages. Future studies could focus on protective factors that promote caregivers' resilience. It is essential to develop psychometrically robust measures for family caregiver burden and gain.
\end{abstract}

Copyright $\odot 2011$ S. Karger AG, Basel
(C) 2011 S. Karger AG, Basel

$1420-8008 / 10 / 0306-0469 \$ 26.00 / 0$

Fax +4161306 1234

E-Mail karger@karger.ch

www.karger.com
Accessible online at: www.karger.com/dem

\section{Introduction}

The World Alzheimer Report estimates that there are currently 35.6 million people with dementia (2010). It is projected that the numbers will double every 20 years, to 65.7 million in 2030 and 115.4 million in 2050 [1]. With increasing life expectancy and declining fertility in many Asian countries, it is expected that the majority of the world's older people will be living in Asia in the 21st century. There will be an $89 \%$ increase in the number of people with dementia in the developed Asia-Pacific countries over the next 20 years, which represents a $10 \%$ increase on what had been published in Lancet in 2005 [1,2].

The problem of family burden in caring for persons with dementia is a common challenge in both developed and developing countries. From 1980 to present, the majority of studies on family care in dementia have focused on understanding the nature and levels of the burden families have in caregiving, particularly how aspects of care are most problematic and detrimental to caregivers. A considerable amount of research literature concerning the stress in caregiving and its effects on caregivers' health has been published in the past decade [3-7].

Different sociocultural factors in different countries may influence a family's commitment to care. Families may have to take full responsibility in taking care of or assist in taking care of the person with dementia to various extents, depending on the available services, resource 
and support to the persons with dementia and their family caregivers. In Asian societies, the family is the first line of support for older persons. Families place great values on filial responsibility. Adult children are expected to take on the responsibilities of caring for their aging parents [8]. For example, in Singapore, close to $95 \%$ of older people co-resided with family members and depended on their family to provide health and instrumental care [9]. As the family is the primary social support system in Asian societies, dementia has a tremendous impact on the whole family. The aim of this paper is to examine the global issues related to family caregiving for older persons with dementia from an Asian perspective.

\section{Methods}

Published empirical studies were reviewed to enable further understanding of caregivers' burden and interventions to help these caregivers. A literature search was carried out using the 1990 to October 2010 databases of CINAHL, Cochrane Library, EMBASE, Google Scholar, PubMed/PsycINFO and SCOPUS. Search terms included 'dementia', 'family caregiving', 'family caregivers', 'burden', 'stress', 'Asian' and 'Asian culture'. The reference lists of identified papers were also searched. The title and abstracts were reviewed. Papers were included if they were peerreviewed, published in English and focused on family caregiving issues for persons with dementia in Asian regions. The following presents a narrative review of the literature.

\section{Caregiver Burden}

It is common that family caregivers receive little support in low- and middle-income countries [1] for their work of caring for persons with dementia. Residential care and community social care systems (home care) are scarce. Governmental policies in these countries also do not provide financial support for the tasks of care provided by the family caregivers. As these caregivers struggle to meet the demands from their own work, family and caregiving, their own physical and emotional health is often ignored. Having no or insufficient personal, financial and emotional resources, many caregivers often experience tremendous stress [10]. Caregiver burden has been defined as a negative reaction to the impact of providing care on caregivers' social, occupational and personal roles [11]. In recent years, the view of caregiving burden has been broadened to involve the physical, psychological, social and financial problems experienced by families caring for a relative with a chronic or mental illness. This view has been accepted by the global community [10].
Some authors distinguish between 'objective' and 'subjective' burden of the family caregiver. Objective burden relates to the caregiver's physical symptoms, behavior and sociodemographic characteristics, as well as factors such as changes in household routine, family or social relations, work, leisure time, and physical health. Subjective burden is the mental distress among family members [12]. Papastavrou et al. [6] further describes that caregiving burden is a multidimensional phenomenon that consists of 4 major domains of care: the framework of care (characteristics of the caregiver, type of the dyadic relationship and availability of social support); the stressful situations of caregiving, which may be primary (such as the problematic behavior of the patient) or secondary (such as other commitments of the caregiver); factors that moderate the perception of stress (such as coping strategies and management of care); and the consequences of caregiving for the general well-being of the caregiver.

There is evidence that chronic stress exposure in caregivers of persons with dementia is associated with physical health decline, psychiatric morbidity and poor quality of life [13]. Chronic stresses may result in poor standard of care, neglect or even abuse of the person with dementia [11]. A study showed that $68 \%$ of caregivers $(n=172)$ were highly burdened (measured by the Burden Interview) and $65 \%$ exhibited depressive symptoms. Women caregivers had higher scores for burden and depression and suffered from social or relational deprivation [6]. A study of Hong Kong Chinese family caregivers of people with Alzheimer's disease using qualitative interviews found that they suffered from heavy psychosocial, financial and physical burdens [14]. A Taiwan study, using the grounded theory approach, found that caregivers' stress often continues after the person with dementia has been institutionalized. Instead of giving up their roles as primary caregivers, the family caregivers become care assistants and establish collaborative relationships with the nursing home staff [15].

It is well established that the symptoms of dementia are an important source of caregiving burden and stress, which in turn is a major determinant of institutionalization for persons with dementia $[6,16]$. Studies also found behavioral problems had a far greater impact on caregivers' lives than cognitive or functional impairment of the person with dementia $[6,16]$. A Singapore study, using the General Health Questionnaire, found that the impact on family caregivers of Chinese patients with dementia correlated significantly with duration of care, presence of delusion, hallucination, depression, insomnia, incontinence and agitation, and behavioral problems [7]. Simi- 
larly, a Korean study found wandering behavior had a negative impact on caregivers' burden [17].

The quality of the relationship preceding the onset of dementia might also have a bearing on the caregiver's situation [18]. The quality of the current relationship between the caregiver and the patient may have an influence on caregiver burden $[16,18]$. A study showed that close kinship to the person with dementia usually imposes a heavy burden [16]. The amount of caregiving time each week and impaired sense of own identity predicted the caregivers' perceived burden as caregivers feel that they are being trapped in their caring role $[16,18]$. However, another study found that caregivers who have low levels of intimacy, communication and affection predicted higher levels of burden [18].

The caregiver's age also has a direct impact on caregiver burden [17]. Gender of the caregivers may have play a role in the caregivers' burden. Globally, almost $80 \%$ of the caregivers are women. They could be the mother, wife or daughter of the person with dementia [2, 10, 19-21]. A study found being a female caregiver predicts a higher level of burden [18]. Other studies show that middle-aged and older women who provided care for an ill or disabled spouse were almost 6 times more likely to have depressive or anxious symptoms as were those who had no caregiving responsibilities [10]. A comparative study on caregivers in 24 centers in India, China and South-East Asia found most caregivers were women and lived with the person with dementia in extended family households. Larger households were associated with lower caregiver strain [21]. Evidently, a challenge in dementia care in the Asian regions is the need to have an in-depth understanding of the concerns of female caregivers and to develop ways to support them.

With the worldwide aging population, it is anticipated that more family caregivers will be involved in the future, and more males are likely to become caregivers. Taking on the caregiving role could mean a role reversal, a change in traditional gender roles within the family in many cultures [10]. A study found that husbands, being the caregivers' of their wives suffering from dementia, expressed anger, worry, weariness, guilt, distress and isolation [22]. At present, there is a paucity of research on the male caregiving role in Asian countries. Little is known about the number and the condition of male caregivers. Additional research needs to be conducted to see how the male caregiving role impacts their lives.

Various instruments have been used for assessing and monitoring the degree of caregiver burden. The 10/66 Dementia Research Group has adopted the Zarit Burden
Interview $(\mathrm{ZBI})$ in a 24-center study in various parts of the world, including Asia [21]. The ZBI has been validated in Hong Kong Chinese and found to be a valid and reliable tool [23]. The Family Caregiving Burden Inventory [24] has also been validated and used in Asian studies and found to be a valid and reliable tool $[3,25]$. The Screen for Caregiver Burden is another instrument that has been used in family caregiver studies [26]. To gain a better understanding of family caregiver burden as well as comparing studies across cultures, it is desirable to have a standardized instrument that can measure family caregiver burden. The ZBI appears to be an appropriate instrument for this purpose.

\section{Conceptualization of Dementia}

The burden of dementia care giving has been explored within the framework of the general stress theories. Caregiver burden is hypothesized as being an acute reaction to providing care that arises as new care demands are introduced or existing care demands intensify. When care demands become increasingly challenging, caregivers respond by employing strategies to meet care demands and decrease the burden of providing care. Caregivers who are unable to adapt or modify their strategies to meet care demands experience burden [11]. However, research has pointed to differences between Eastern and Western conceptualization of dementia and expected coping behaviors, which have implications for caregivers' perceived burden, problem recognition, help seeking, service engagement and service delivery [26].

Culture gives a framework to individuals for interpreting and giving meaning to personal experiences. It affects how the family assigns meaning to dementia [26]. For example, a study on India or the Indian origin of Hindi- and Punjabi-speaking caregivers found they perceived symptoms of dementia as partly resulting from lack of effort by the persons themselves and possibly lack of family care. Therefore, they believed that people should overcome their own problems and family action might be part of the solution [27].

A study on a multiethnic sample of Asian-American caregivers in the United States [28] found that caregivers adopted different models in the conceptualization of dementia: a biomedical model, a folk model and a mixedfolk model. The biomedical model emphasized abnormal brain-based disorders. Caregivers referred to the conditions as a disease and used biomedical labels such as dementia and Alzheimer's disease. In the folk model, de- 
mentia-related changes were simply viewed as 'normal' consequence of growing old by some caregivers. For example, Vietnamese caregivers used the label 'confused' and viewed this as an expected part of the aging process. A Chinese caregiver understood his mother's condition as 'memory deterioration' and attributed this to multiple causes, including normal old age, mental illness, having a 'difficult personality', and not being 'open-minded' enough. In the mixed-folk model, caregivers used biomedical labels, such as Alzheimer's disease, but drew upon folk models of illness to explain the illness, such as Alzheimer's disease was due to psychosocial stress (e.g. loss of a loved one, a tragic event) or to the process of 'normal' aging.

Asian countries like China, Japan and Korea are strongly influenced by Confucianism, Buddhism and Taoism. Mok et al. [29] found that Chinese people in Hong Kong hold the belief that whatever happens is heaven's will and that they accept it. Some people see the cause of dementia as related to destiny sent from heaven for their benefit. They accept the illness, in the same way as they accept other responsibilities. This acceptance is strongly influenced by beliefs of 'tian (heaven) ming (destiny)' which means following the nature of the universe and the principles of how things work. When a person follows 'tian', they will be blessed; thus, it is unwise not to follow 'tian'.

These cultural conceptualizations may help some caregivers to accept and endure the existence of illness or hardship and accept these as a normal course of life or fate. These beliefs may also help the family caregivers avoid denial or guilt which are frequently mentioned in Western literature [30]. It may offer some protective effects on the family. However, these beliefs may prevent family members from engaging in active help-seeking behavior, and act as a barrier to diagnosis and intervention $[28,31]$. Effective communication between healthcare professionals and families is essential for eliciting their understanding of dementia. A full understanding of the specific way that culture could influence the burdens of family caregivers and the ways that these burdens are expressed in relation to caring for a relative with dementia may play an important part in the development of a holistic model for family-centered care.

\section{Coping with the Task of Caregiving}

Different strategies have been used by family caregivers to cope with their caregiving challenges. For example, avoidance-coping may represent a risk factor associated with higher level of stress in family caregivers of persons with dementia [32]. Lazarus' classification of coping strategies, problem-solving and emotion-focused strategies, are frequently mentioned in studies related to family caregivers' coping [22]. Higher scores on the burden scale were found to be associated with use of emotionfocused coping strategies, while less burdened relatives used more problem-solving approaches to care-giving demands [6]. However, some Asian researchers questioned the validity of Lazarus' dichotomous classification of coping strategies for Chinese subjects. They suggest that coping strategies should not be viewed as 2 discrete categories as all coping strategies have both problem-focused and emotion-focused properties. Thus, these properties could be seen as independent dimensions which can both be applied to the description of any coping strategies [33].

Coping may not simply be a response to stress. Antonovsky [34] argued that the way people interpret events is crucial for whether they will cope with a situation successfully or will become stressed by it. A study in Thailand found that families perceived caring as suffering. Influenced by Buddhism, suffering is considered to be part of life: people do not only experience happiness, but also suffering as well. Thus, people do not need to be afraid of suffering; they must accept it [31]. A Singaporean study found religion and spirituality has a positive effect on coping for family caregivers of persons with dementia [35]. As mentioned above, the study by Mok et al. [29] found that Chinese people, influenced by their culture and religion, accept the responsibility of taking care of a family member with dementia as a normal course of life or fate.

Antonovsky [34] proposed the concept of 'sense of coherence' (SOC). SOC is a general and relatively stable coping resource, influencing how a person will cope with stressful events and threats, and it is assumed to be positively connected to health, including mental health [36]. Antonovsky developed a 29-item SOC scale to measure the SOC construct. Another study found that family caregivers of persons with dementia who had high SOC had a better perceived quality of life and less depressive symptoms [37]. Similarly, the study by Andrén and Elmståhl [38] found caregivers with lower burden reported significantly better perceived health and had a higher mean score of SOC than caregivers with higher burden.

The concept of SOC has been studied in the Asian context. A Taiwan study found family caregivers who have a better SOC have less depressive symptoms [39]. A study of Asian-American female family caregivers who were 
Chinese and Filipino found that a better SOC might contribute to positive health outcomes [40]. These findings concur with those studies conducted in Caucasian populations. Therefore, the assessment of caregivers' burden and SOC is important for health care professionals to understand the caregivers' situation. Family caregivers could be helped to reflect upon their coping strategies to find balance in the situation.

Various instruments have been developed to assess SOC. Apart from Antonovsky's SOC scale, a Singapore study has used the Gain in Alzheimer's Care Instrument to measure SOC [41]. A study in Japan has developed a 3 -item SOC measure for a population survey. The different instruments used in SOC make it difficult to compare findings across studies. For this reason, it is desirable to develop a standardized instrument to measure family caregivers' SOC across cultures [42].

The relationship between the family caregiver and the person with dementia may have an influence on caregiver burden. Expressed emotion (EE) is a construct that has been used for the past 3 decades to describe family members' criticism, hostility and emotional involvement with a mentally ill relative within the context of family caregiving [43]. In Western countries, $\mathrm{EE}$ has been well accepted to be a generalized risk factor associated with caregiver stress in different mental health problems such as schizophrenia, depression and dementia [44]. For example, Tarrier et al. [45] found high EE was associated with higher scores of caregiver distress and strain. In the Asian context, a Japanese study using the Camberwell Family Interview Schedule (CFI) [46] found that there was a tendency for lower expression of both positive and negative emotional reactions towards the family member with dementia [47].

As the Camberwell Family Interview Schedule requires intensive training in its administration and coding because of the complexity in analyzing the interview data, Chien and Chan [43] validated the Level of Expressed Emotion (LEE), a self-administered questionnaire, in the Hong Kong Chinese population. The 50item Chinese version of the LEE has been found to be satisfactory in assessing changes in family functioning in family caregivers of persons with psychotic disorders. Chien and Chan commented that though the concept of EE originated from Western studies, it seems to be relevant for Chinese culture. There are similarities between Chinese and Western cultures in the constructs of EE. For example, Chinese people also believe that excessive emotion is harmful to an individual's physical and mental health and that people should keep their emotions un-

Family Caregivers' Burden: The Asian Perspective der control to maintain family functions and relationships [43]. To date, there have been only limited studies on EE in Asian family caregivers of persons with dementia. Further investigations are needed to gain more conclusive evidence.

Culture may also have an influence on the expression of needs and help-seeking behavior of family members caring for a relative with dementia. Caudle [48] found that Latin-American families place particular emphasis on encouraging open expression of needs and feelings. On the contrary, in Asian countries, there is a belief in keeping secret something unfortunate or degrading to the family name [49]. The Chinese view the family as the basic and most important unit of human life. They make clear distinctions between 'ijiren' (insider, the family) and 'wairen' (outsider, those not belong to the family). Family secrets should be confined to insiders and not be revealed to outsiders. The fear of reporting unfavorable things about oneself might prevent family caregivers from discussing their difficulties with people outside their family, thus preventing them from seeking help. Another factor that prevents the family from seeking help may be related to the fear that the dementia sufferer will be labeled 'mentally ill', which is socially stigmatized [49]. Gilbert et al. [26] further argued that the shame and stigma of mental health problems, and accessing services, are related with 'discovery' and confidentiality. For example, some Asians have fears that any sensitive information about mental states and difficulties they might share with healthcare professionals will be revealed to others [26]. Thus, they are reluctant to use mental health services. Understanding caregivers' unique beliefs which are shaped by sociocultural backgrounds will assist healthcare professionals in developing strategies to promote better coping with caregiving difficulties.

Social support is considered to be of great value in families who experience the burden associated with caring for an individual with dementia. The study by Tremont et al. [50] found caregivers with high levels of burden reported greater family dysfunction. More family support was positively related to higher levels of family functioning [51]. A study on Chinese family caregivers found informal support correlates strongly with psychological well-being. The emotional support provided by spouses and children was found to have a significant correlation with enhanced life satisfaction and decreased depressive symptoms in the caregivers. Social support may enhance self-efficacy of the caregivers, thus reducing depressive symptoms [52]. Social support was also found to be the best predictor of family caregiver burden in an- 
other Asian study [53]. Thus, strengthening the families' social network may represent a useful strategy to alleviate care burden of the family.

Caregiving has traditionally been perceived as an extremely stressful process. This can be criticized as focusing only on the negative aspects of caring. Nolen [19] comments that the dominance of the stress-burden model in family services has meant that interventions have focused almost exclusively on stress reduction with little attention to more expanded range outcomes. Recently, Folkman's revised stress and coping model explains that both positive psychological appraisals and burdens can be experienced concurrently by family caregivers $[41,54]$. The bidirectional congruence caregiving model also views caregiving as a mutual meeting of needs between the caregiver and care recipient. This model further provides explanation on why some caregivers adapt to their caregiving roles better than others [55].

Providing care to a family member with dementia can bring about positive outcomes such as personal growth, mastery, improved relationships and renewed perspectives on life [56]. A Singapore study found that caregiver gain was significantly associated with the mental wellbeing of the caregiver, use of active management as a caregiving strategy, and participation in caregiver education and support group programs. Thus, caregiver interventions should target these factors [41].

Yap et al. [57] developed a 10-item instrument questionnaire, the Gain in Alzheimer Care Instrument (GAIN), based on a qualitative study conducted to investigate the experience of gain in family caregivers of people with dementia so as to measure caregiving gains. The psychometric properties of GAIN were examined in Singapore on family caregivers of persons with dementia and found to be a reliable and valid instrument [57]. Future studies may further test GAIN in other sociocultural settings and to explore the structure of its factors.

Studies have found protective factors that could mediate or moderate caregiver burden [58-61]. A systematic review found finding meaning in caregiving and caregiver motivation to provide care has a positive impact on caregiver well-being [58]. Some Asian studies found other factors. An Australian family caregiver study identified that hopefulness in the caregiver as a crucial part of the coping process in long-term coping with a family member with a mental illness. Families drew their hopefulness from both formal and informal supports, as well as from within and without [59]. A study in Japan found that persons with dementia who were satisified with daily life and the ability to perform tasks had a strong impact on reducing the sense of burden felt by the caregivers. The study suggested that providing support that would enhance the quality of life of the persons with dementia could indirectly help to alleviate the burden felt by family members caring for them [60]. Future studies need to explore the positive aspects of caregiving, such as the caregiver gain, protective factors that could mediate or moderate caregiver burden, and ways to promote caregivers' resilience [61].

The majority of the reviewed studies in this paper were cross-sectional. Dementia is a progressive disease, and caregivers may experience different levels of burden at different stages of the illness process. There is a need for longitudinal studies to have a better understanding of the family caregivers' burdens and needs at different illness stages in different regions and cultures.

\section{Family Interventions}

In many Asia countries, the family is the primary long-term caregiver and an important resource for persons with dementia. The focus of care for persons with dementia has shifted from the sick person to family-oriented care, with family members being partners in care. There is a growing amount of empirical evidence supporting that some intervention strategies have a beneficial impact on the burden of family caregivers [62]. For example, a training program for caregiving skills has been found to be relatively more effective in reducing family distress and strengthening care management $[63$, 64]. Training programs, such as on the behavioral management technique, cognitive stimulation, prosthetic memory aids, and general problem solving have been used to help caregivers deal with behavioral problems of people with dementia. Other types of training programs that have been found effective focus on strengthening family caregivers' assertive and cognitive skills in dealing with the negative thoughts of people with dementia, coping with loss and grief, increasing enjoyable activities, and better use of supporting resources [63].

The Resources for Enhancing Alzheimer's Caregiver Health (REACH) are multicomponent caregiver interventions involving active provision of information, didactic instruction, role playing, problem solving, skills training, stress management and telephone support groups. A multisite trial on Hispanic, Latino and Caucasian caregivers found that the intervention group experienced significant greater improvement in quality of life and lower prevalence of clinical depression than the control group [65]. 
A review of empirical studies on family intervention for caregivers of people with dementia found studies conducted in Asian regions. Four studies were conducted in Hong Kong. An experimental study compared the effectiveness of providing a mutual support group program for Hong Kong Chinese family caregivers of a relative with dementia with that of the conventional family service available to this group. Results showed the mutual support group had greater improvement in distress levels and quality of life when compared with the control group [66]. Another randomized controlled trial evaluating a case management model for people with mild dementia did not show significant effects in reducing caregiver burden, but encouraged family caregivers to seek external support [67]. A more recent trial tested the effectiveness of a nurse-led dementia family psychoeducation program on caregivers' and health outcome of people with dementia. The 6-month intervention included having a nurse as the case manager for the family caregiver and the person with dementia. Twelve group psychoeducation sessions ( $2 \mathrm{~h}$ each) were conducted, which aimed at equipping caregivers with caregiving skills, improving their ability to cope and enhancing mutual support among members. The results of the study showed that the intervention group had significantly greater improvements in symptoms and institutionalization rates of people with dementia, as well as on caregivers' quality of life and burden over the 15-month follow-up period, compared with the routine care group as controls. This study supported that a psychoeducation program can improve the psychosocial functioning of Hong Kong Chinese persons with dementia and their caregivers [3]. Another study found that cognitive-behavioral programs could be effective in improving the resourcefulness of Chinese caregivers [68]. When comparing the outcomes of these trials, the nurseled dementia family psychoeducation program appears promising. The limitations of these studies, however, were that single-centered trials with small number of samples.

In India, a randomized controlled trial was conducted using the 10/66 Dementia Research Group caregiver brief intervention which was developed to be delivered at home using existing local resources. This study found significant improvement in caregiver mental health and distress due to behavioral disturbances. However, there was no significant effect on caregiver burden and the behavioral problems in the persons with dementia [69].

A study in Thailand provided a 6-month group counseling intervention in an out-patient setting for caregivers. The results suggested improvement in the neuropsy-

Family Caregivers' Burden: The Asian Perspective chiatric symptoms of persons with dementia when compared with the control group. However, this study did not measure the burden of caregivers [70].

Few meta-analyses and systematic analyses on the effectiveness of caregiver intervention have been conducted. A meta-analysis of 30 controlled studies identified modest, but significant, benefits in caregiver knowledge and their psychological morbidity, but there was no significant effect on caregiver strains [71]. Another metaanalysis found interventions generally had a small-tomedium effect for most caregivers' outcomes. Involvement of patients and caregivers in a structured program was the only intervention that was found to be statistically significant [72].

A more recent systematic review of studies was conducted on a variety of interventions, including psychoeducation interventions, support, multicomponent interventions, use of exercise or nutrition, case management, and computer-aided support intervention. The review found that psychoeducation interventions (such as programs on behavioral therapy, stress and coping skills, with follow-up periods from 1 to 9 months) had statistically significant effects in improving depression and subjective well-being among caregivers. Multicomponent interventions resulted in statistically significant effects in improving self-efficacy, depression, subjective well-being and perceptions of burden. Noneffective interventions that should be avoided included only referring caregivers to support groups, only providing self-help materials and only offering peer support [62].

Previous studies adopted different interventions, designs and outcome measures. There is still a need for future trials which should have more rigor randomization, follow-up for at least 6 months and use psychometrically robust outcome measures [73]. Further research is necessary to identify effective follow-up strategies. Further trials could also assess outcomes for which there is limited evidence, such as types of exercise programs and costeffectiveness.

In traditional Asian societies, the family is expected to take care of their elders at home. However, socioeconomic and demographic changes have led to changes in family caregiving in modern Asian communities. Nuclear families, which have become widespread, have replaced the traditional extended family living in one house. The majority of women generally have a full-time job. With low fertility rates reported in many Asian countries, less young people will be available in the family to take care of the older persons at home. Yet the older generation are still very dependent on the young for care and support. 
Many older people do not want to stay in old-age homes as they have the belief that staying in an old age home indicates abandonment by one's children [8]. These changes raise serious concerns on the future care of older people with dementia. If the family members are not available to take care of their elders, there have to be alternatives. Examples of alternatives are care homes, day centers, respite care, paid caregivers [1] and home care interventions, such as the Care of Persons with Dementia in their Environment (COPE) Program [74]. There is a need to determine alternatives that will be acceptable to both family caregivers and the persons with dementia.

\section{Conclusion}

This paper examines the global problem of family caregiver burden for older persons with dementia from an Asian perspective. Family caregiver burden is a multidimensional phenomenon, involving their mental health, physical condition, social life, financial status and functioning of the family as a whole. It is not limited to any particular society or culture. There is a need to take into account the unique beliefs which are shaped by sociocultural backgrounds to understand caregivers' experiences and coping reactions. This will assist healthcare professionals in developing strategies to reduce caregiver burden and promote better coping with caregiving difficulties.

With socioeconomical and demographical changes, families need support when taking on caregiving roles. In many Asian countries, families assume an important role in taking care of and supporting their older relatives. Thus, the government has the responsibility of establishing policies and services to support these family caregivers. Evidence-based interventions, such as caregiver psychoeducation, support and respite care should be routinely provided. For instances where the family cannot carry out the traditional caregiving role, there is a need to develop alternatives of care that are acceptable to people with dementia and the families.

Further trials could assess outcomes of interventions for which there is limited evidence. There is a need for future trials with more rigor randomization and followup for at least 6 months. There is also a need for longitudinal studies to assess family caregiver burdens and needs at different illness stages. Future studies could focus on protective factors that promote resilience in caregivers. Studies could also focus on developing standardized psychometrically robust measures that can be used across cultures to measure caregiver burden and gains.

\section{References}

1 Alzheimer's Disease International: World Alzheimer Report 2010: The Global Economic Impact of Dementia. Alzheimer's Disease International, London, 2010.

2 World Health Organization: Alzheimer's disease: the brain killer, 2006. http://www. searo.who.int/en/Section1174/Section1199/ Section1567/Section1823_8066.htm (accessed July 10, 2010).

3 Chien WT, Chan SWC: A controlled trial of a nurse-led psychoeducation program for families of people with dementia. Hong Kong Ment Health J 2009;35:29-42.

$\checkmark 4$ Etters L, Goodall D, Harrison BE: Caregiver burden among dementia patient caregivers: a review of the literature. J Am Acad Nurse Pract 2008;20:423-428.

5 Tally R, Crew JE: Framing the public health of caregiving. Am J Public Health 2007:97: 224-228.

-6 Papastavrou E, Kalokerinou A, Papacostas SS, Tsangari H, Sourtzi P: Caring for a relative with dementia: family caregiver burden. J Adv Nurs 2007;58:446-457.
Kua EH, Tan SL: Stress of caregivers of dementia patients in the Singapore Chinese family. Int J Geriatr Psychiatry 1997;12:466469.

8 Chan SWC, Chiu HFK, Chien WT, Goggins W, Thompson D, Hong B: Predictors of change in health-related quality of life among older people with depression - a longitudinal study. Int Psychogeriatr 2009;21: 1171-1179.

-9 Mehta KK: Stress among family caregivers of old persons in Singapore. J Cross Cult Gerontol 2005;20:319-334.

10 World Federation of Mental Health: Caring for the Caregiver: Why Your Mental Health Matters When You Are Caring for Others. WFMH, Woodbridge, 2010.

11 Given CW, Given BA, Stommel V, Azzouz F: The impact of new demands for assistance on caregiver depression: tests using an inception cohort. Gerontologist 1999;39:76-85.

12 Reine G, Lancon C, Simeon MC, Duplan S, Auquier P: Caregiver burden in relatives of persons with schizophrenia: an overview of measure instruments. Encephale 2003;29: 137-147.
13 Mahoney R, Regan C, Katona C, Livingston $\mathrm{G}$ : Anxiety and depression in family caregivers of people with Alzheimer disease: the LASER-AD study. Am J Geriatr Psychiatry 2005; 13:795-801.

$14 \mathrm{Ng}$ P, Ho WC: Experience in coping with Alzheimer's disease at home: a study of Chinese family caregivers. J Soc Work Disabil Rehabil 2005;4:1-14.

15 Lau WY, Shyu YI, Lin LC, Yang PS: Institutionalized elders with dementia: collaboration between family caregivers and nursing home staff in Taiwan. J Clin Nurs 2008; 17 : 482-490.

16 Annerstedt L, Elmståhl S, Ingvad B, Samuelsson SM: An analysis of the caregiver's burden and the 'breaking-point' when home care becomes inadequate. Scand J Public Health 2000;28:23-31.

17 Lim YM, Son G, Song J, Beattie E: Factors affecting burden of family caregivers of community dwelling ambulatory elders with dementia in Korea. Arch Psychiatr Nurs 2008; 22:226-234. 
18 Campbell P, Wright J, Oyebode J, Job D, Crome P, Bentham P, Jones L, Lendon C: Determinants of burden in those who care for someone with dementia. Int J Geriatr Psychiatry 2008;23:1078-1085.

$\checkmark 19$ Nolan M: Supporting family carers in the UK: overview of issues and challenges. $\mathrm{Br} \mathrm{J}$ Nurs 2001;10:608-613.

20 Ng GT: Singapore: characteristics of family caregivers and care recipients and their caregiving experiences. Hallym Int J Aging 2008; 10:41-62.

21 Prince M: Care arrangements for people with dementia in developing countries. Int J Geriatr Psychiatry 2004:19:170-177.

-22 Samuelsson AM, Annerstedt L, Elmståhl S, Samuelsson SM, Grafstrőm M: Burden of responsibility experienced by family caregivers of elderly dementia sufferers. Scand J Caring Sci 2001;15:25-33.

23 Chan TSF, Lam LCW, Chiu HFK: Validation of the Chinese version of the Zarit Burden Interview. Hong Kong J Psychiatry 2005:15: 9-13.

24 Novak RG, Guest C: Application of a multidimensional Caregivers Burden Inventory. Gerontologist 1989:29:798-803.

25 Chou KR, Jiann-Chyun L, Chu H: The reliability and validity of the Chinese version of the Caregiver Burden Inventory. Nurs Res 2002:51:324-331

26 Gilbert P, Bhundia R, Mitra R, McEwan K, Irons $\mathrm{C}$, Sanghera J: Cultural differences in shame-focused attitudes towards mental health problems in Asian and non-Asian student women. Ment Health Relig Cult 2007; 10:127-141.

-27 Fontaine JL, Ahuja J, Bradbury NM, Philips S, Oyebode JR: Understanding dementia amongst people in minority ethnic and cultural groups. J Adv Nurs 2007;60:605-614.

-28 Hinton L, Franz CE, Yeo G, Levkoff SE: Conceptions of dementia in a multi-ethnic sample of family caregivers. J Am Geriatr Soc 2005;53:1405-1410.

-29 Mok E, Lai CKY, Wong FLF, Wan P: Living with early-stage dementia: the perspective of older Chinese people. J Adv Nurs 2007;59: 591-600.

-30 Wong M, Chan S: The coping experience of a group of Chinese parents with children diagnosed of childhood cancer - a qualitative study-research in brief. J Clin Nurs 2005; 14: 648-649.

-31 Sethabouppha H, Kane C: Caring for the seriously mentally ill in Thailand: Buddhist family caregiving. Arch Psychiatr Nurs 2005; 19:44-57.

- 32 Di Mattei VE, Prunas A, Novella L, Marcone A, Cappa SF, Sarno L: The burden of distress in caregivers of elderly demented patients and its relationship with coping strategies. Neurol Sci 2008:383-389.
33 Phillips MR; Pearson V: Coping in Chinese communities: the need for a new research agenda; in Bond $\mathrm{MH}$ (ed): The Handbook of Chinese Psychology. Hong Kong, Oxford University Press, 1996, chap 28.

34 Antonovsky A: The structure and properties of the sense of coherence scale. Soc Sci Med $1993 ; 725-733$.

35 Lim J, Griva K, Goh J, Chionh HL, Yap P: Coping strategies influence caregiver outcomes among Asian family caregivers of persons with dementia in Singapore. Alzheimer Dis Assoc Disord 2010, E-pub ahead of print.

36 Schnyder U, Buchi S, Sensky T, Klaghofer R: Antonovsky's sense of coherence: trait or state? Psychother Psychosom 2000;69:296302.

37 Vilimaki TH, Vehvilainen-Julkunen KM, Pietila AK, Pirttila TA: Caregiver depression is associated with a low sense of coherence and health-related quality of life. Aging Ment Health 2009;13:799-807.

-38 Andrén S, Elmståhl S: The relationship between caregiver burden, caregivers' perceived health and their sense of coherence in caring for elders with dementia. J Clin Nurs 2008;17:790-799.

39 Tang ST, Li CY: The important role of coherence in relation to depressive symptoms for Taiwanese family caregivers of cancer patients at the end of life. J Psychosom Res 2008:64:195-203.

40 Jones PS, Zhang XE, Jaceldo-Siegl K, Meleis $\mathrm{Al}$ : Caregiving between two cultures: an integrative experience. J Transcult Nurs 2002; 13:202-209.

-41 Liew TM, Luo N, Ng WY, Chionh HL, Goh J: Predicting gains in dementia caregiving. Dement Geriatr Cogn Disord 2010:29:115-122.

42 Togari T, Yamazaki Y, Nakayama K, Shimizu J: Development of a short version of the sense of coherence scale for population survey. J Epidemiol Comm Health 2007:61:921-922.

43 Chien WT, Chan SWC: Further validation of the Chinese version of the Level of Expressed Emotion Scale for research and clinical use. Int J Nurs Stud 2010:47:190-204.

44 Götell E, Brown S, Ekman SL: The influence of caregiver singing and background music on vocally expressed emotions and moods in dementia care: a qualitative analysis. Int J Nurs Stud 2009:46:422-430.

45 Tarrier N, Barrowclough C, Ward J, Donaldson C, Burns A, Gregg L: Expressed emotion and attributions in the carers of patients with Alzheimer's disease: the effect on carer burden. J Abnorm Psychol 2002:111:340-349.

46 Brown GW, Monck EM, Carstairs GM, Wing JK: Influences of family life on the course of schizophrenic illness. Br J Prev Soc Med 1962:16:55-68

47 Nomura H, Inoue S, Kamimura N, Shimodera S, Mino Y, Gregg L, Tarrier N: A cross-cultural study on expressed emotion in carers of people with dementia and schizophrenia: Japan and England. Soc Psychiatry Psychiatr Epidemiol 2005;40:564-570.
48 Caudle P: Providing culturally sensitive health care to Hispanic clients. Nurse Pract 1993;18:40-51.

49 Tseng WS, Lu QY, Yin PY: Psychotherapy for the Chinese: culture considerations; in Lin TY, Tseng WS, Yeh EK (eds): Chinese Societies and Mental Health. Hong Kong, Oxford University Press, 1995, pp 281-294.

50 Tremont G, Davis JD, Bishop DS: Unique contribution of family functioning in caregivers of patients with mild to moderate dementia. Dement Geriatr Cogn Disord 2006: 21:170-174.

51 Saunders JC: Family functioning in families providing care for family members with schizophrenia. Issues Ment Health Nurs 1999;20:95-113.

52 Au A, Lai M, Lau K, Pan P, Lam L, Thompson L, Gallagher-Thompson D: Social support and well-being in dementia family caregivers: the mediating role of self-efficacy. Aging Ment Health 2009;13:761-768.

53 Chien WT, Chan S, Morrissey J: The perceived burden among Chinese family caregivers of people with schizophrenia. J Clin Nurs 2007; 16:1151-1161

54 Folkman S: Positive psychological states and coping with severe stress. Soc Sci Med 1997; 45:1207-1221.

55 Kahana E, Young R: Clarifying the caregiving paradigm: challenges for the future; in Biegel DE, Blum A (eds): Ageing and Caregiving: Theory, Research and Policy. Newbury Park, Sage, 1990, pp 76-97.

56 Sanders S: Is the glass half empty or half full? Reflection on strain and gain in caregivers of individuals with Alzheimer's disease. Soc Work Health Care 2005;40:57-73.

- 57 Yap P, Luo N, Ng WY, Chionh HL, Goh B: Gain in Alzheimer Care Instrument - a new scale to measure caregiving gains in dementia. Am J Geriatr Psychiatry 2010;18:68-76.

58 Quinn C, Clare L, Woods R: The impact of motivations and meanings on the wellbeing of caregivers of people with dementia: a systematic review. Int Psychogeriatr 2010;22: 43-55.

59 Bland R, Darlington Y: The nature and sources of hope: perspectives of family caregivers of people with serious mental illness. Perspect Psychiatr Care 2002;38:61-68.

60 Fujino N, Okamura H: Factors affecting the sense of burden felt by family members caring for patients with mental illness. Arch Psychiatr Nurs 2009;23:128-137.

61 Hilgeman MM, Allen RS, DeCoster J, Burgio LD: Positive aspects of caregiving as a moderator treatment outcome over 12 months. Psychol Aging 2007;22:361-371.

62 Parker D, Mills S, Abbey J: Effectiveness of interventions that assist caregivers to support people with dementia living in the community: a systematic review. Int J Evid Based Healthc 2008;6:137-172. 
63 Acton G, Winter MA: Interventions for family members caring for an elder with dementia; in Fitzpatrick JJ, Stewart BJ, Lyons LK (eds): Annual Review of Nursing Research. New York, Springer, 2002, vol 20, pp 149179.

-64 Ostwald SK, Hepburn KW, Caron W, Burns T, Mantell R: Reducing caregiver burden: a randomised psycho-educational intervention for caregivers of persons with dementia. Gerontologist 1999;39:299-309.

65 Elliott AF, Burgio LD, Decoster J: Enhancing caregiver health: findings from the resources for enhancing Alzheimer's caregiver health II intervention. J Am Geriatr Soc 2010:58: 30-37.

66 Fung W, Chien W: The effectiveness of a mutual support group for family caregivers of a relative with dementia. Arch Psychiatr Nurs 2002;16:134-144.
67 Lam LCW, Lee JSW, Chung JCC, Lau A, Woo J, Kwok TCY: A randomized controlled trial to examine the effectiveness of case management model for community dwelling older persons with mild dementia in Hong Kong. Int J Geriatr Psychiatry 2010;25:395-402.

68 Au A, Lee K, Leung P, Pan PC, Thompson L, Gallagher-Thompson D: The coping with caregiving group program for Chinese caregivers of patients with Alzheimer's disease in Hong Kong. Patient Educ Couns 2010;78: 256-260.

69 Dias A, Dewey ME, D’Souza J, Dhume R, Motghare DD, Shaji KS, Menon R, Prince M, Patel V: The effectiveness of a home care program for supporting caregivers of persons with dementia in developing countries: a randomised controlled trial from Goa, India. PLoS One 2008;4:3:e2333.

70 Senanarong V, Jamjumras P, Harmphadungkit K, Klubwongs M, Udomphanthurak S, Poungvarin N, Vannasaeng S, Cummings JL: A counselling intervention for caregivers: effect on neuropsychiatric symptons. Int J Geriatr Psychiatry 2004;19:781-788.
71 Brodaty H, Green A, Koschera A: Meta-analysis of psychosocial interventions for caregivers of people with dementia. J Am Geriatr Soc 2003;51:657-664.

-72 Pinquart M, Sörensen A: Helping caregivers of persons with dementia: which interventions work and how large are their effects? Int Psychogeriatr 2006; 18:577-595.

73 Gavrilova SI, Ferri CP, Mikhaylova N, Sokolova O, Banerjee S, Prince M: Helping carers to care - the 10/66 Dementia Research Group's randomized control trial of a caregiver intervention in Russia. Int J Geriatr Psychiatry 2009:24:347-354.

74 Gitlin LN, Winter L, Dennis MP, Hodgson N, Hauck WW: A biobehavioral home-based intervention and the well-being of patients with dementia and their caregivers: the COPE randomized trial. JAMA 2010:304: 983-991. 